\title{
Knowledge Sharing in E-Collaboration
}

\author{
Neil Ireson and Gregoire Burel \\ University of Sheffield, UK
}

\begin{abstract}
For eCollaboration to be effective, especially where it attempts to promote true collective decision-making, it is necessary to consider how knowledge is shared. The paper examines the knowledge sharing literature from the perspective of eCollaboration and discusses the critical challenges, principally the motivation of knowledge sources and maintenance of semantics, and describes how techniques and technologies can be employed to alleviate the difficulties. The paper concludes with an example of how such technologies are being applied for Emergency Response, to facilitate knowledge sharing both amongst the citizens and between the citizens and organisations.
\end{abstract}

Keywords: eCollaboration, knowledge sharing, emergency response.

\section{Introduction}

Knowledge sharing is an activity where agents (individuals, communities or organisations) exchange their knowledge (information, skills or expertise). It is intrinsically linked to the knowledge management process, which can be broadly characterised by four activities, the: creation, storage and retrieval, transfer and application of knowledge. Whilst knowledge sharing is fundamentally concerned with the transfer activity, it cannot be isolated from the other activities: the complete sharing process involves the externalisation of their knowledge by the source (often referred to as knowledge capture), the transmission of that knowledge, and finally the internalisation of the knowledge by the recipient. Therefore any methodology concerning knowledge sharing must also consider the wider knowledge management activities.

The majority of the knowledge sharing research and practice has focused on the sharing within and between professional organisations, however more recently there has been an increasing realisation that organisations can exploit the potential benefits offered through harnessing the power of the users to which they provide products and services. The catalyst for this new model of interaction between organisation and users (i.e. customers of businesses, citizens or serviceusers of public bodies) has been the development of Web 2.0 technologies, such as: online social-networking, content-sharing, wikis and blogs.

The traditional interaction model, whereby any information provided by users is anonymously absorbed into the organisation, and organisations broadcast to all users regardless of individual needs, is giving way to a complex web of interactions, where information relevant to the organisation is communicated amongst 
individuals and groups, and between those individuals and groups and the organisation itself. The most common form of benefit to be derived from users is via user-feedback on the products/services provided by organisations, either as simple ratings or more qualitative assessment. However it is also possible that, rather than users just providing a reaction to the organisations activities, they can be pro-actively engage in the decision-making processes that determine how an organisation's activities are carried out. Users can act as information-gathers, determining the nature, extent and importance of issues and opportunities faced by the organisation. For example, The Ben \& Jerry's Facebook page 11 has nearly 1 million fans who have created new flavours, determined by interactive polls, Johnson \& Johnson's set up the Baby Center2, the Webs number one global interactive parenting network, where they can engage their consumers and curate conversations to solicit information on given topics, and possibly most famously the use of collaborative technologies during the Obama presidential campaign [1].

In essence organisations utilising technology that makes it easy for like-minded individuals to connect and collaborate around the topics (both personal to professional) they care about. However there are inherent issues in ensuring that this more direct engagement of users satisfies both organisation and citizen requirements. Organisations require accurate, coherent, objective information, upon which to base their decision-making, however the subjective and conversational nature of citizens' input means: it can contain personal references; stated facts may be speculative, incomplete or simply incorrect; some citizens may even seek to provide deliberately misleading information; and, as organisations do not control the content of user communication, much of the information will not be of interest to the organisation. Therefore the organisations need to employ technologies to translate the copious quantities of unstructured conversational data into accurate, coherent, objective, structured information: in effect, to determine the signal from the noise. In addition, if organisations are to truly engage in a two-way dialogue with users then it is necessary for them to determine the information that is of interest to the users. To maintain an effective relationship with users, organisations should respect users' limited attention by minimising the amount of irrelevant information they communicated.

Organisations wishing to take advantage of their users are therefore forming a new model of knowledge; rather than explicitly being held within an organisation, knowledge is seen as a systemic property of people in "communities of practice", i.e. groups with shared interests who will benefit from collaboration and sharing knowledge. Information is not only held within formal documents and systems, but also in dialogues amongst groups of people. In this view, knowledge is both individual and collective, and it has crucial implications for an organisation's knowledge management practices. In this context, communities of practice generally, and knowledge-building communities specifically, form the arena for the creation and dissemination of knowledge.

\footnotetext{
1 http://www.facebook.com/benjerry Accessed 14/12/09.

2 http://community.babycenter.com/ Accessed 14/12/09.
} 
The majority of the work which considers knowledge sharing in eGovernment does so from the perspective of either intra- or inter- organisational sharing, or how such technologies can improve the communication from organisations to citizens, Vitvar et al 2] provides a collection of recent work in these areas. The potential benefits which can be derived from the use of user-generated content in eGovernment has been recognised [34, however the work neither systematically considered the issues in terms of the knowledge sharing literature nor implements and evaluates the suggested solutions.

\section{Knowledge Sharing Context}

In order to develop the correct knowledge sharing methodology, and technologies, to apply in a given scenario it is necessary to consider the application context. In eCollaboration, as the requirements upon the degree of engagement increase: from information gathering through consultation to systems which attempt to promote true collaboration and collective decision-making, the challenges upon efficacious knowledge sharing will increase. In Cummings' extensive survey of the literature on knowledge sharing [5] he identifies five primary contexts that can affect knowledge sharing, these are:

- Relational — The relationship between the source and the recipient, i.e. their distance in: physical, social, cultural, educational, etc. terms.

- Knowledge - Assessed accordingly to: explicitness, i.e. the extent to which knowledge is able to be expressed, codified and transmitted through in formal language; and embeddedness, i.e. the extent to which it is possible to isolate the package of knowledge to be shared, or whether that knowledge is embedded within the source (people, products, tools, routines, etc.).

- Recipient - Ability of to successful receive and internalise the shared knowledge, determine by their: motivation, learning capacities, intent, knowledge experience, collaborative experience, retentive capacity and culture.

- Source - The sources knowledge-sharing capability, i.e. the ability to explicitly communicate credible and comprehensible knowledge.

- Environmental - The broader environment in which the sharing occurs, which has a vicarious effect upon the impact of the other contexts.

Applications which facilitate eCollaboration are fundamentally focused on exploiting the ability to share knowledge between organisations and their community of users, as well as within the community of users. As the target community expands and users become more diverse, the relational context of the sources and recipients weakens. The complexity of the knowledge will vary according to the degree to which the user is allowed, and expected, to express their mind: from simple ratings provided in petitions through questionnaires to open dialogues where users can freely express their thoughts, feelings, experience and opinions. Where knowledge is contained within such conversational data it is necessary to consider the extent to which the knowledge is embedded within the dialogue, in order to ensure that it is shared as an interpretable package. It should also 
be noted that when packaging (codifying) the knowledge it is possible to over codify the knowledge, for example if imperfection in the knowledge exist at the source this should not be hidden by codification. Also there may be a trade-off between the recipients ability to consider knowledge in its fully codified form and their need for timely knowledge, this is particularly likely if the recipients context is such that they have limited resources to spend on sharing activities.

\section{Motivation for Knowledge Sharing}

To develop a effective knowledge sharing system it is essential to consider the motivation of the users to share their knowledge. In fact, a user-centred design philosophy may argue that unless the technology employed fosters users' motivations, or at least does not actively hinder them, it is unlikely to function effectively. Broadly there are two types of motivation: intrinsic (self-oriented motives) - self expression, personal development, utilitarian motives, economic motives and knowledge efficacy; and extrinsic (external-oriented motives): social affiliation, enhance reputation, social ranking, competition, reciprocity, expected economic and organisational rewards.

There are a myriad of factors, both personal traits and the characteristics of the task, which influence any individual's motivations to act, and a single individual's motivations will vary over time. Studies into group dynamics tend to indicate that each member of the group takes into account what others are doing [6], and have done [7], before deciding to act (i.e. they are extrinsically motivate). Although other research argues that rational actors will contribute if their efforts are cost-effective, that is, if they know (or think they know) they can "make a difference", then they will contribute regardless of others in the group [8]. This self-efficacy, or belief that one's actions have an effect, does seem to motivate sharing in online environments 910 and has been indicated as an important factor to consider in the decision to participate in a democratic process [1]. Although there will not be a single type of motivation for all users of an eCollaboration system, in organisations there is an expectation of some extrinsic reward for any knowledge shared, whilst in community-based systems, incentives become less significant thus the intrinsic motivation to participate and share knowledge becomes more important [12].

\section{Knowledge Sharing in a Community of Practice}

As was stated in the introduction, eCollaboration is primarily concerned with facilitating knowledge sharing in Communities of Practice $(\mathrm{CoP})$, where a $\mathrm{CoP}$ can be characterised as an informal network of individuals who share a common set of information needs or problems. The challenge is to support such communities and make them effective; provided them with knowledge management tools to allow more natural, intuitive and efficacious access to knowledge. In order to consider the systematic factors which influence knowledge generation and sharing in CoP, the C4P framework is adopted 13. This posits that knowledge 
is generated and shared when there is purposeful conversation around content in context. $\mathrm{C} 4 \mathrm{P}$ is shorthand for content, conversation, connections, (information) context, and purpose. These elements comprise a non-linear system that occurs in a CoP. It is assumed that the more these elements are present in any community, the more likely and effective the knowledge generation and sharing will be. The following sections will provide a critical assessment the C4P elements, in terms of how they impact upon a knowledge-sharing community. This allows the focusing of context and motivation factors towards more pragmatic considerations of an eCollaboration system.

\subsection{Content}

Content, i.e. the contributed information: text, images, videos, etc, satisfies a number of purposes in a $\mathrm{CoP}$, it: is an explicit container for the information (and knowledge) available; is an asset to attract member by providing immediate value; defines, implicitly, the domain of interest; and provides the basis for conversations. Whilst it is desirable to build a system which solicits members to provide content, quality and not quantity is the key measure, i.e. content must be relevant, accurate and coherent. Certain extrinsic rewards, such as: social interaction ties, reciprocity and identification, increase an individual's quantity of shared knowledge but not necessarily its quality [14, and in fact may have a negative impact [15].

The generation of quality content is one of the fundamental challenges of eCollaboration, and is obviously a prerequisite to having effective knowledge sharing. It is generally the case that the minority of community members provide the majority of content, for example in Wikipedia $2.5 \%$ of registered users contributed $80 \%$ of all the content [16. There have been numerous studies into how to motivate quality content contributions, including those specific to online communities. Whilst the research does not result in a clear "best practice" list of techniques to employ in all circumstances, the most important factors are generally given as: age, socio-economic status, gender, proficiency and familiarity 17. It is therefore necessary to consider the nature of the users to determine the likely interaction with the system the users will exhibit, in order to maximise the quality of their contribution.

Research shows that one of the key ways to stimulate content contribution is to receive feedback (i.e. replies, reviews, ranking), however it also points out that feedback is more likely if the contributor is engaged with the community [18 19], this leads to difficulties in soliciting contributions from newcomers. It is therefore beneficial, especially for newcomers, that knowledge capture services guide uses to generate messages which are more likely to stimulate feedback, i.e. ones which are short, on-topic, asking questions and using less complex language. In addition it is advisable for newcomers to introducing themselves to the community via autobiographical testimonials [18]. Newcomers are also unwilling to contribute if the do not fully understand how to use the technology [19, therefore the system should be simple to learn and use, it is also necessary to ensure suitable 
access is provided, as people can choose (perceived) ease of access over content quantity/quality when selecting an information source [20].

\subsection{Conversation}

The conversations in the system are the primary means by which knowledge is shared. Where conversation which is focused on a piece of quality content is likely to build upon the knowledge embedded with it, and as long as the content is relevant to the CoP purpose the conversation is likely to be as well. If there is a strong and clear sense of shared purpose then it becomes more likely that everyone involved understands that the goal of every conversation is to support that purpose, and not change the topic or thread. The challenge is to provide such focused conversations that draw out meaningful knowledge (signal), rather than aimless chat (noise). However not all conversation will be directly toward the purpose of the $\mathrm{CoP}$, and therefore it may be necessary for organisations to guide them, for example by asking for clarification or providing further information about of points of interest. Providing such feedback, including even being given specific and challenging goals, has been shown to stimulate individuals contribution [17.

To facilitate sharing it is necessary to provide support for the nature of online conversation. The individual needs to maintain continuity and comprehension of the flow of a conversation even when it is disjointed. Generally this is achieved by archiving the conversion threads (as with forums and blogs), however when revisiting conversations users' comprehension is aided by allowing the focusing on specific user (or group of users), time or spatially related input.

\subsection{Connections}

Connections (which can be seen as a strong relational context) foster the development of trust and common goals. Whilst previous work which suggests that developing such connections requires rich interpersonal interactions and a shared history 21, recent studies have shown that even with impersonal sharing processes users perceived their actions as being a social act [15. Individuals may be willing to share their personal knowledge due to strong feelings toward the virtual community, without necessarily trusting other members in the virtual community [14]. Therefore, rather than connections amongst individuals, it is the connection to the community as a whole which may be just as influential on the motivation to share knowledge.

In addition whilst connections build trust, trust does not necessarily impact upon of knowledge sharing, it is arguable that trust is not crucial unless individuals have a degree of risk (possible cost) in sharing knowledge. One cost which can cause a hesitation to contribute is the fear of criticism, or of misleading the community members (not being sure that their contributions are important, or completely accurate, or relevant to a specific discussion), therefore members must feel safe from personal attack 22, which can be provided by moderation, either by an assigned moderator or by the community as a whole. 


\subsection{Information Context}

Information context is the who, what, where, when, why, and how that facilitates the interpretation of the knowledge by the recipient and thus enables them to determine the relative merits of the knowledge to them and their situation, and eases its internalisation and reuse. It should also be emphasised that knowledge sharing is not content sharing, rather it is the ability to share the knowledge embedded within content which the technology aims to facilitate. If the context of the information within the content is relatively explicit, or the sender and receiver share a conceptual understanding of the content, then sharing the content may be sufficient to also share its embedded knowledge. However if this is not the case addition information (context) must also be provided with the content. It can consist of information about the knowledge provider, or the knowledge itself, e.g. links to related material and previous uses of the knowledge.

\subsection{Purpose}

It could be said that the shared purpose of a $\mathrm{CoP}$ is its defining characteristic, and that the community content, conversations, connections and context are both guided by and defined by its purpose. The community may well have some explicitly stated purpose (or vision) and ideally it's actual purpose, the one defined by the communities members and their actions and interactions, will be congruent with that: as a shared purpose is seen to promote quality sharing, and also reduce the quantity of off-topic exchanges [14]. The a key to the success of knowledge sharing in a $\mathrm{CoP}$ is that personal purpose should match the group purpose [23. One of the main determinants of both quantity and quality of knowledge sharing appears to be the expectations of successful community-related goals [9], however if these conflict with an individual's personal goals then that can have a negative impact upon the quantity of knowledge sharing [14].

\section{Technological Support for Knowledge Sharing}

An organisation's need for knowledge management and sharing tools that foster collaboration becomes even more important when the required information is outside of organisations: within the wider community. Knowledge tools can enable or reduce the time taken in searching, browsing and interpreting documents to find out how they are related to one another and thus locate the similarities and differences among pieces of information. Exposing the implicit information structures can allow otherwise isolated information to be placed into a meaningful context and thus help users manage information (and knowledge) more efficiently. The recent technologies employed to facilitate knowledge sharing in eCollaboration can be broadly encapsulated by the Web 2.0 technologies, that is, those technologies used by: forums, blogs, new feeds, wikis, etc. This section considers how such technologies are employed to aid the knowledge sharing 
activity and how incorporating Semantic Web technologies (sometimes termed Web 3.0) can be used to further enhance the effectiveness of Web 2.0 technologies for knowledge sharing.

Web 2.0 technologies primarily improve knowledge sharing by: lowering temporal and spatial barriers between the knowledge source and recipient; providing some degree of dialogue management between a potential multitude of sources and recipients; and improving access to information, i.e. easing the storage, retrieval and transfer of knowledge artefacts, i.e. the documents (notes, emails, images, etc.) which implicitly or explicitly contain the knowledge. However simply providing the ability for this interaction to take place has limited value if it ignores when and how the quality of knowledge sharing will be enhanced [23. Whilst Web 2.0 technologies can aid communities in the construction of a collaborative information pool, the information it contains can be of varying quality and have multifarious expressions. This leads to difficulties when attempting to share the knowledge and develop a collective understanding, especially when the community is made up from diverse individuals, with different: cultural backgrounds, education, experience, expertise, etc. where there is an increased likelihood of a variety of expressions and terminology which require some degree of tacit knowledge to interpret the meaning.

In terms of the $\mathrm{C} 4 \mathrm{P}$ framework discussed above Web 2.0 technologies readily enhance the ability to: store, retrieve and transfer content; facilitate conversation between temporally and spatially disparate groups of individuals; and managed the network of links between connected individuals. However they do not inherently provide means to store, retrieve and transfer the context of the information, and without this such technologies provide means for information exchange and social interaction leading to content rather than knowledge sharing. In order for knowledge sharing to take place, and the community to develop a collective understanding, the technologies should also provide a means for the knowledge, which resides in its member and is implicit in the content they share, to be made explicit, to ensure that the intended meaning (semantics) of the knowledge is maintained. Existing solutions to this problem can be classified into three main approaches 24]: internalisation, socialisation and externalisation.

With internalisation approaches the shared information context is contained within the community dialogues: held in the blogs, mailing lists, bulletin boards, discussion forums, etc. This presumes that the information context is expressed by community members, which requires a community motivated towards question answering. Relying on such approaches means there is no structure or standard in how the information context is expressed and thus retrieval of the context is potential difficult and arduous, thus useful knowledge can easily be lost. The socialisation approach aims at supporting the sharing of knowledge through linking of individuals likely to share an understanding. Thus, the technology provides some shared space where contextually related users (e.g. concerned with the same issue or working on same task) can exchange knowledge and engage with each other. The externalisation approaches aim to develop an explicit model of shared 
conceptualisations, known as an ontology, which provide a formal description of concepts and the relationships between them.

Ontologies provide an abstract, simplified (possibly incomplete) view of a domain and therefore a means to explicitly represent the context of information: modelling the semantics (meaning) of information in a way both processable by computers and usable for the communication of meaning between human users. They have been identified as particularly applicable to addressing the issues, discussed above, which effect sharing in CoP, such as: missing or imperfect conceptual models, unclear system boundaries, and heterogeneous representations 25. Ontologies provide the ability to organise knowledge into a more controllable form and can be used to determine the relative importance, context, significance and association between pieces of information. This can enable CoP engaged in tasks with ad hoc interactions, between diverse individuals, to organise the knowledge artefacts into the predefined conceptual classes of the ontology, allowing more efficacious access to knowledge.

\section{E-Collaboration Ontology}

This section briefly describes the key concepts of an eCollaboration ontology called CURIO (Collaborative User Resource Interaction Ontology) 3 . In effect it represents the world view of eCollaboration information and provides a pragmatic combination of the formal ontologies used for describing the knowledge areas. A full description of the Ontology is beyond the scope of this paper (however the full specification is available on the website), the following briefly describes the five key concepts:

Resource: The ontology is articulated around the concept of Resource, which can be defined as an abstract piece of information or anything that can be inserted in the system by a user. The ontology distinguishes two main Resource types that are the focal concepts containing the information of primary interest to the users, Document and Thread, see below. Each Resource can possess a number of properties, such as: a title, a date of creation, a description, a creator, a set of tags, etc. Additionally, each Resource may be subscribed by any user of the system through the use of a ResourceFeed, this mechanism allows a user to be notified when a resource changes. In addition a Resource can be associated with a localisation, which provides temporal and spatial information.

Document: Documents are any media (i.e. text, images or audio) content which is provided by the user, they are pieces of information that can be: used as evidence to induce, describe and define a Thread; associated to Users (as a creator or modifier), and assigned a number of Tags.

Thread: A Thread acts as a container describing how resources are combined into coherent structures, e.g. Events or Arguments. Thus all the information (resources and users) related to a given Thread are linked with an instance of

3 http://purl.org/net/curio/ns\# Accessed 14/12/09. 
a Thread. In addition threads are composed into structures to express their interrelationships, this composition can be a simple subsumption (i.e. a global thread with a number of sub-threads) or may involve more complex relationships based on causality and correlation.

User: The representation of a user includes the ability to express their personal details, preferences and profile their interests.

Tag/Comment/Rating: Tags can be either free-text or related to some tag hierarchy (taxonomy or ontology) that provides a stable, dereferencable identifier for the concept expressed by the Tag. In addition to tagging, it is possible to attach a (textual) Comment and (numeric) Rating to a Resource. Such concepts are key to providing the ability to effective retrieve information via browsing and searching.

\section{Knowledge Sharing in Emergency Response}

The techniques and technologies described above have be applied in developing an Emergency Response (ER) system that combines organisation and citizen information to improve situational awareness and thus the decision-making process. The nature of such an eCollaboration application means the citizens engaged in the system may not have a strong relational context, simply being brought together by happenstance. Initially the requirements for both the organisational professionals were gathered and compared, to ensure these are satisfied, in general there was a correlation between the information needs of these two groups, although citizens expressed a stronger desire for social information and professionals had more strict non-functional requirements. Both groups basically expressed a need for factual (temporal, spatial and topic related) information 26. In the ER domain there is also a need for timeliness of information in order to react to critical incidents as quickly as possible.

The system allows users can log in with federated identities, such as OpenID 4 , which allows new uses to import their personal information, thus can enabling newcomer introduction. In addition a user profile is used to direct their attention to content which is likely to be of interest. As users add content (Documents) into the system they receive automatic suggestions to tag (or locate) their content, encouraging them to provide a more systematic encoding of their content. The uploaded information is then automatically organised into Threads (incidents/events) which can be validated and augmented by the ER professionals. This process of combining evidence provides reinforcement to increase confidence in the citizens' information. Users can add further information to Threads and also subscribed to them, to receive notification of changes and thus promote further contributions.

The information in the system can be accessed via the ontological concepts, the information can be viewed on maps and timelines and can be filtered according to user type (i.e. citizen or professional), or the associated tags, descriptions

\footnotetext{
${ }^{4}$ http://openid.net/ Accessed 14/12/09.
} 
and comments. Therefore it is possible to develop an overall situational awareness as the incident/event information is added to the system and to focus in on particular information (in a locality or related to a given topic) which is of interest to the user. The evaluation of the initial prototype with both citizens and ER professional indicates that such codification of knowledge improves the ability to quickly and accurately evaluate the information available in assessing the location and severity of incidents.

\section{Conclusions}

In order for effective eCollaboration it is necessary to consider how knowledge can be effectively shared, both amongst citizens and between those citizens and organisations. This task poses challenging difficulties, as the individuals involved may: be from diverse backgrounds; have no previous relationship; lack common understanding; provide varying quality input; and have disparate information needs. By examining the literature in the context of eCollaboration two critical factors emerge: the need to motivate users to contribute their knowledge and need to codify that knowledge to enable its interpretation and reuse. To address these issues the use of Web 2.0 and Web 3.0 techniques is proposed, and shown to provide potential benefit in the development of situational awareness for Emergency Response.

Acknowledgments. This work has been supported by the European Commission as part of the project WeKnowIt (FP7-215453).

\section{References}

1. Kes-Erkul, A., Erkul, R.E.: Web 2.0 in the process of e-participation: The case of organizing for america and the obama administration. Working Paper 09-001, National Center for Digital Government (October 2009)

2. Vitvar, T., Peristeras, V., Tarabanis, K. (eds.): Semantic Technologies for E-Government. Springer, Heidelberg (2010)

3. Krogstie, J.: Citizens, from consumers to prosumers: e-government services typologies revisited. In: Proceedings of the NordicCHI'06 Workshop User Involvement and representation in e-Government projects (2006)

4. Zappen, J.P., Harrison, T.M., Watson, D.: A new paradigm for designing e-government: web 2.0 and experience design. In: Proceedings of the 2008 international conference on Digital government research, pp. 17-26 (2008)

5. Cummings, J.: Knowledge sharing: A review of the literature. Technical report, The World Bank Operations Evaluation Department (2003)

6. Granovetter, M.S.: The strength of weak ties. The American Journal of Sociology 78(6), 1360-1380 (1973)

7. Macy, M.W.: Chains of cooperation: Threshold effects in collective action. American Sociological Review 56(6), 730-747 (1991)

8. Oliver, P.E., Marwell, G.: The paradox of group size in collective action: A theory of the critical mass. ii. American Sociological Review 53(1), 1-8 (1988) 
9. Benbunan-Fich, R., Koufaris, M.: Motivations and contribution behaviour in social bookmarking systems: An empirical investigation. Electron. Market. 18(2), 150-160 (2008)

10. Chen, I.Y.L., Chen, N.S.: Kinshuk: Examining the factors influencing participants knowledge sharing behavior in virtual learning communities. Educational Technology and Society 12(1), 134-148 (2009)

11. Cruickshank, P., Smith, C.F.: Self-efficacy as a factor in the evaluation of e-petitions. In: Parycek, A. (ed.) Proceedings of EDEM 2009 - Conference on Electronic Democracy, pp. 223-232. Austrian Computer Society, Vienna (2009); Written as part of the EuroPetition project

12. Palmisano, J.: A motivational model of knowledge sharing. In: Burstein, F., Holsapple, C.W. (eds.) Handbook on Decision Support Systems, vol. 1, pp. 355-370. Springer, Heidelberg (2008)

13. Hoadley, C.M., Kilner, P.G.: Using technology to transform communities of practice into knowledge-building communities. SIGGROUP Bull. 25(1), 31-40 (2005)

14. Chiu, C.M., Hsu, M.H., Wang, E.T.G.: Understanding knowledge sharing in virtual communities: an integration of social capital and social cognitive theories. Decis. Support Syst. 42(3), 1872-1888 (2006)

15. Peddibhotla, N.B., Subramani, M.R.: Contributing to public document repositories: A critical mass theory perspective. Organization Studies 28(3), 327-346 (2007)

16. Tapscott, D., Williams, A.D.: Wikinomics: How Mass Collaboration Changes Everything. Portfolio Hardcover (2006)

17. Beenen, G., Ling, K., Wang, X., Chang, K., Frankowski, D., Resnick, P., Kraut, R.E.: Using social psychology to motivate contributions to online communities. In: CSCW '04: Proceedings of the 2004 ACM conference on Computer supported cooperative work, pp. 212-221. ACM, New York (2004)

18. Arguello, J., Butler, B.S., Joyce, E., Kraut, R., Ling, K.S., Rosé, C., Wang, X.: Talk to me: foundations for successful individual-group interactions in online communities. In: CHI '06: Proceedings of the SIGCHI conference on Human Factors in computing systems, pp. 959-968. ACM, New York (2006)

19. Burke, M., Marlow, C., Lento, T.: Feed me: motivating newcomer contribution in social network sites. In: CHI '09: Proceedings of the 27th international conference on Human factors in computing systems, pp. 945-954. ACM, New York (2009)

20. Mann, T.: Library research methods: a guide to classification, cataloging and computers. Oxford University Press, New York (1993)

21. Nahapiet, J., Ghoshal, S.: Social capital, intellectual capital, and the organizational advantage. Academy of management review, 242-266 (1998)

22. Ardichvili, A., Page, V., Wentling, T.: Motivation and barriers to participation in virtual knowledge-sharing communities of practice. Knowledge Management 7(1), 64-77 (2003)

23. Hendriks, P.: Why share knowledge? the influence of ict on the motivation for knowledge sharing. Knowledge and Process Management 6, 91-100 (1999)

24. Novak, J., Wurst, M.: Supporting knowledge creation and sharing in communities based on mapping implicit knowledge. Journal of Universal Computer Science 10(3), 235-251 (2004)

25. Stuckenschmidt, H., van Harmelen, F.: Information Sharing on the Semantic Web. Springer, Heidelberg (2004)

26. Lanfranchi, V., Ireson, N.: User requirements for a collective intelligence emergency response system. In: Proceedings of 23rd BCS HCI Group Conference (HCI 2009), September 2009. British Computer Society, Cambridge (2009) 